\title{
Scattering and absorption properties of biomaterials for dental restorative applications
}

\author{
A. Fernández-Oliveras \\ alilia@ugr.es
}

\section{Rubiño}

M. M. Pérez

\author{
Departamento de Óptica, Facultad de Ciencias, Universidad de Granada, Campus Fuentenueva s/n. \\ 18071 Granada, Spain \\ Departamento de Óptica, Facultad de Ciencias, Universidad de Granada, Campus Fuentenueva s/n. \\ 18071 Granada, Spain \\ Departamento de Óptica, Facultad de Ciencias, Universidad de Granada, Campus Fuentenueva s/n. \\ 18071 Granada, Spain
}

The physical understanding of the optical properties of dental biomaterials is mandatory for their final success in restorative applications. Light propagation in biological media is characterized by the absorption coefficient, the scattering coefficient, the scattering phase function, the refractive index, and the surface conditions (roughness). We have employed the inverse adding-doubling (IAD) method to combine transmittance and reflectance measurements performed using an integrating-sphere setup with the results of the previous scattering-anisotropy goniometric measurements. This has led to the determination of the absorption and the scattering coefficients. The aim was to optically characterize two different dental-resin composites (nanocomposite and hybrid) and one type of zirconia ceramic, and comparatively study them. The experimental procedure was conducted under repeatability conditions of measurement in order to determine the uncertainty associated to the optical properties of the biomaterials. Spectral variations of the refraction index and the scattering anisotropy factor were also considered. The whole experimental procedure fulfilled all the necessary requirements to provide optical-property values with lower associated uncertainties. The effective transport coefficient presented a similar spectral behavior for the two composites but completely different for the zirconia ceramic. The results demonstrated that the scattering anisotropy exerted a clearly distinct impact on the optical properties of the zirconia ceramic compared with those of the dental-resin composites.

[DOI: http://dx.doi.org/10.2971/jeos.2013.13056]

Keywords: Optical properties, absorption coefficient, scattering coefficient, reflectance and transmittance measurements, inverse addingdoubling algorithm, dental restorative biomaterials

\section{INTRODUCTION}

Optical properties of biological media are key parameters in such contrasting fields as the food industry [1] and legal or forensic medicine [2]. In particular, research concerning the interaction of laser light with new biomaterials is of great interest for clinical applications. The development of laserbased techniques for treatments and diagnostics depends on the knowledge of light distribution in the biological media. In restorative dentistry this is especially important, since novel mechanisms for improving patients' comfort and satisfaction are permanently sought.

On the one hand, the most disturbing aspects for dentistry patients are related to the use of mechanical instruments. Therefore, the development of light tools is an appealing alternative [3]. Low-power lasers are used mainly because of their biostimulatory, analgesic, and anti-inflammatory action. Highpower lasers which produce visible physical effects are used as substitutes for scalpel or conventional rotary devices (e.g. laser-ablation equipment instead of a bur or turbine handpiece) [4]. There are also some laser varieties that are replacing halogen lamps, for the same purposes: curing and whitening [5]. The use of other laser types has been proposed as a method of decontaminating treated areas in order to prevent caries and plaque as well as to detain bacterial spread [6, 7]. The improvement in the adhesion of restorative material is also an advantage of laser treatment cited by clinicians [8].

On the other hand, aesthetic failure is one of the most widespread reasons for restoration replacement. Aesthetic restoration involves a visible match of the optical properties of restorative material and natural teeth. These optical properties are determined by absorption and scattering of light emerging on the surface and inside the medium. Among the main appearance properties of restorative materials, the perceived color and translucency are intimately related with light-scattering properties [9, 10]. Thus, the final success and quality of the restoration imply a thorough physical understanding of light interaction in dental biomaterials.

The interaction of light in an absorbing and scattering medium can be estimated using the radiative transport equation which may be solved using the adding-doubling method [11]-[13]. Based on the latest one, the inverse addingdoubling (IAD) method was proposed [14] and implemented in the iad program [15]. With this technique, reflection and transmission measurements performed with an integrating 
sphere are converted to the optical properties of the media (scattering and absorption coefficients). This program has been broadly used to determine the absorption and scattering properties of diverse biological media [16]-[21]. Measuring the diffuse reflection, collimated transmission $\left(T_{c}\right)$ and total transmission $\left(T_{t}=T+T_{c}\right)$ allows the program to determine the three key optical properties: absorption coefficient $\left(\mu_{a}\right)$, scattering coefficient $\left(\mu_{s}\right)$, and anisotropy coefficient $(g)$. However, if only the diffuse reflection and total transmission measurements are available, then only the absorption and the reduced scattering $\left(\mu_{s}^{\prime}\right)$ coefficients can be calculated. Thus, in these cases, additional measurements are required in order to determine the scattering anisotropy factor. This is an important point, since materials with the same reduced scattering coefficient but different $g$ values can show different angular scattered intensities, both in form and magnitude. Therefore, using the absorption and the reduced scattering coefficients alone for comparing materials or for reconstruction using inverse methods based, for example, on diffusion theory would lead to incorrect interpretations [22].

Currently, dental-resin composites and dental zirconia ceramics are two biomaterials which are used to replace the most important dental tissues in irreversibly diseased teeth, i.e. enamel and dentine, respectively. Dental nano-filled resin composites have recently been introduced [23]. Nanotechnology applied to dental composites has provided filler particles that are dramatically smaller, can be dispersed in high concentrations and are polymerized into the resin system with molecules designed to be compatible when coupled with a polymer. Since this molecular manufacturing provides other unique characteristics, scattering and absorption properties of nanocomposites could differ when compared with those of traditional composites. In this work, we studied two dentalresin composites that presented two different types of inorganic filler (classified as nano and hybrid) with a similar organic matrix (polymeric).

Yttrium cation-doped tetragonal zirconia polycrystal (3YTZP) has been improved and progressively more widely employed in restorative dentistry with the aim of avoiding the infrastructure of metallic prostheses. The microstructure of 3Y-TZP ceramics consists of small equiaxed grains with diameter sizes depending on the sintering temperature [24]. The sintered ceramic for dental applications is fabricated using computer-aided design and manufacturing (CAD/CAM) procedures from pre-sintered zirconia ceramic. This material arose as a versatile and promising biomaterial because of its good mechanical and biological properties $[25,26]$. However, optical characterizations have not been completely developed for this material. In this sense, scattering and absorption studies in dental zirconia ceramics become necessary. Our objective is to experimentally analyze the scattering and absorption properties of two different types of dental-resin composites and one type of zirconia ceramic and comparatively study them. With this aim, the IAD method was applied for these biomaterials, combining previous results of goniometric measurements with transmission and reflection measurements performed using a laser-integrating-spherebased setup. For comparisons between the different types of materials, the uncertainties associated to the optical properties were also determined.

\section{THEORETICAL BACKGROUND}

In this work, dental biomaterials were considered to have homogeneous optical properties and a uniform refraction index. This ensured that light will travel in a straight line until scattered or absorbed. The geometry of the samples was approximated by a plane-parallel slab of finite thickness. Such a shape allows generalization to layered samples or extension to an infinitely thick sample. The boundaries were assumed to be smooth and to reflect specularly according to Fresnel's law. The distribution of light was assumed to be static with time (both optical properties which change and irradiance times shorter than about one nanosecond were excluded) and it was assumed that there were no light sources in the material. The last assumption was that the polarization of light may be ignored.

In addition to the thickness, light propagation through a slab is determined by three parameters: the absorption coefficient, the scattering coefficient, and the phase function. The phase function indicates the fraction of light scattered from an obstacle as a function of the scattering angle. If normalized, the phase function represents the angle's probability of being the angle between the direction of the photons before and after the scattering event. The phase function of several dental biomaterials was experimentally analyzed and discussed in detail elsewhere [27]-[29].

Like biological tissues, biomaterials are assumed to be random turbid media, with variations in the optical properties small enough to prevent localized absorption. In other words, they are considered to have volumetric scattering and absorption properties rather than being composed of discrete scattering and absorption centers distributed in a non-scattering, non-absorbing medium. Volumetric absorption (or scattering) is determined by multiplying an absorption (or scattering) cross section with the density of absorbers (or scatterers) [30]. This is how the absorption coefficient, $\mu_{a}$, and scattering coefficient, $\mu_{s}$, are defined. These coefficients represent the probability per infinitesimal path length that a photon will be absorbed or scattered by the material, correspondingly. The scattering and absorption coefficients are typically measured in inverse millimeters, and the reciprocal of these coefficients is the average distance that light will travel before being scattered or absorbed, respectively.

The behavior of light in a biomaterial sample for which the geometry can be approximated by a plane-parallel slab can be described by the radiative transport equation [31]

$$
(\hat{s} \cdot \bar{\nabla}) L(\bar{r}, \hat{s})=-\mu_{t} L(\bar{r}, \hat{s})+\mu_{s} \int_{4 \pi} p\left(\hat{s}, \hat{s}^{\prime}\right) L(\bar{r}, \hat{s}) d \omega^{\prime}
$$

where $L$ is the radiance $\left(\mathrm{Wcm}^{-2} \mathrm{sr}^{-1}\right)$ at position $r$ in the direction of the unit vector $s$. The differential solid angle $d \omega^{\prime}$ has the unit vector $\mathrm{s}$ as an outward normal. The phase function $p$ represents the fraction of light scattered from the direction $s^{\prime}$ in the direction $s$ and is normalized such that it is unity when 
integrated over all directions. The attenuation or transport coefficient, $\mu_{t}$ is defined by the sum of scattering and absorption coefficients

$$
\mu_{t}=\mu_{a}+\mu_{s}
$$

which express the exponential attenuation of light with depth in the material (the probability that light will travel in the material to a depth $z$ without scattering or absorbing is $e^{-\mu_{t} z}$ ). The reciprocal of the transport coefficient is the average distance that light will travel before being scattered or absorbed, usually called the mean free path or penetration depth (since it is a measure of how deep light can penetrate the material), $l_{t}$ :

$$
l_{t}=\frac{1}{\mu_{t}}
$$

The left-hand side of the radiative transport equation, Eq. (1), describes the rate of change of the intensity at the point indicated by $r$ in the direction of the unit vector $s$. This rate of change is equal to the intensity lost due to absorption and scattering (the first term on the right-hand side) plus the intensity gained through light scattering from all other directions into the direction $s$ (the last term on the right-hand side).

Calculations of light distribution based on the radiative transport equation require the knowledge of the absorption coefficient, the scattering coefficient, and the phase function. For these parameters to be determined, a solution of the transport equation is needed first. Due to its difficulty, diverse approximations are made to solve the radiative transport equation. The diffusion approximation is based on the observation that the light distribution in highly scattering media tends to become isotropic. This is true even if the initial light-source distribution and the phase function are highly anisotropic. Each scattering event blurs the light distribution and, as a result, the light distribution tends towards uniformity as the number of scattering events increases. In the diffusion theory, the radiance in Eq. (1) can be divided into collimated and diffuse components:

$$
L(\bar{r}, \hat{s})=L_{\text {coll }}(\bar{r}, \hat{s})+L_{d}(\bar{r}, \hat{s})
$$

Intuitively, once light becomes isotropic, only backward scattering terms change the net flux, forward scattering is indistinguishable from no scattering. The collimated radiance, $L_{\text {coll }}$, includes both the light scattered into a direction parallel to the incident beam and any unscattered light. The unscattered light does not interact with the material and satisfies the Beer's law, with transmission equation

$$
T_{c}=e^{-\mu_{t} z}
$$

with $z$ being the material thickness. Because the collimated radiance includes light scattered forwards, the beam is attenuated not by the usual total extinction or transport coefficient, $\mu_{t}$, but by the reduced transport coefficient $\mu_{t}^{\prime}$

$$
\mu_{t}^{\prime}=\mu_{a}+\mu_{s}^{\prime}
$$

where $\mu_{s}^{\prime}$ is the reduced scattering coefficient

$$
\mu_{s}^{\prime}=(1-g) \mu_{s}
$$

and $g$ is the scattering anisotropy factor, which represents the phase function, and is defined by the mean value of the scattering-angle:

$$
g=\int_{-1}^{1} p(\cos \theta) \cos \theta d(\cos \theta)
$$

The scattering anisotropy factor $g$ is a measure of the amount of forward direction retained after a single scattering event: it varies between complete backward scattering $(g=-1)$ and complete forward scattering $(g=1)$. If $g=0$, then the medium is said to be isotropic, signifying that photons have the same probability of going in any direction.

The scattering coefficient is reduced because light scattered in the forward direction is indistinguishable from unscattered light. The fraction of light not scattered within the forward peak is $(1-g)$, and consequently, the scattering coefficient is reduced by a factor of $(1-g)$. Thus the reduced scattering coefficient, $\mu_{s}^{\prime}$, represents the amount of light scattered outside of the collimated portion of the radiance and within the diffuse portion of the radiance. The reduced transport coefficient, $\mu_{t}^{\prime}$, incorporates forward-scattered light into the collimated beam in the same way, such that light was incorporated into expressions for the reduced scattering coefficient. The collimated light is attenuated at a rate proportional to the reduced transport coefficient

$$
\left(\hat{s_{0}} \cdot \bar{\nabla}\right) L_{\text {coll }}(\bar{r}, \hat{s})=-\mu_{t}^{\prime} L_{\text {coll }}(\bar{r}, \hat{s})
$$

where $s_{0}$ is a unit vector in the direction of the incident flux. The amount of collimated light entering the slab is given by

$$
L_{\text {coll }}(\bar{r}, \hat{s})=\left(1-r_{s}\right) L_{i n c}(\bar{r}, \hat{s})
$$

where $L_{i n c}$ is the illuminating flux and $r_{S}$ is the specular reflection coefficient given by the usual Fresnel reflection formula. The solution of Eq. (9), subject to the initial condition of Eq. (10), is

$$
L_{\text {coll }}(\bar{r}, \hat{s})=\left(1-r_{s}\right) L_{i n c}(\bar{r}, \hat{s}) e^{-\mu_{t}^{\prime} z / \mu_{0}}
$$

where $\mu_{0}$ is the cosine of the angle that $s 0$ forms with the $\mathrm{z}$ axis $\left(z / m u_{0}\right.$ is the distance incident light travels in the material to reach a depth $z$ in the slab) and $L_{i n c}$ may be defined from a monodirectional flux $\pi F_{0}(r)$ which illuminated the top surface of the sample [32]

$$
L_{\text {inc }}(\bar{r}, \hat{s})=\pi F_{0}(r) \delta\left(\mu-\mu_{0}\right)
$$

with $\delta$ being the delta function. The diffuse radiance $L_{d}$ includes the light scattered in all the directions except the one parallel to the incident beam and is characterized by the diffusion approximation as a sum of a diffuse radiant fluence $\phi_{d}$ and a diffuse irradiance (radiant flux per unit area) $E_{d}$. These are defined as the first two moments of the radiance $L_{d}$ :

$$
\begin{gathered}
\phi_{d}(\bar{r})=\int_{4 \pi} L_{d}\left(\bar{r}, \hat{s}^{\prime}\right) d \omega^{\prime} \\
\bar{E}_{d}(\bar{r})=\int_{4 \pi} L_{d}\left(\bar{r}, \hat{s}^{\prime}\right) \hat{s}^{\prime} d \omega^{\prime}
\end{gathered}
$$

The diffuse radiance can then be expressed as

$$
L_{d}(\bar{r}, \hat{s})=\frac{1}{4 \pi} \phi_{d}(\bar{r})+\frac{3}{4 \pi} \bar{E}_{d}(\bar{r}) \cdot \hat{s}
$$


where the factors of $1 / 4 \pi$ and $3 / 4 \pi$ result from normalization. Eq. (15) represents the first two terms of the Taylor expansion for the diffuse radiance $L_{d}$, where $\phi_{d}$ represents the isotropic and $E_{d}$ the anisotropic (slightly forward directed) contribution to the diffuse radiance. The diffusion approximation simplifies the sum to the first two terms in order to have a more tractable transport equation [33]. Specifically, if we substitute this two-term expansion of the radiance into the radiative transport equation and then integrate over $\omega$, the result is [30]

$$
\bar{\nabla} \phi_{d}(\bar{r})=-3 \mu_{t}^{\prime} \bar{E}(\bar{r})+\bar{Q}_{1}(\bar{r})
$$

where $Q_{1}$ is the first-order source term of the volumetric source $Q$ :

$$
\bar{Q}_{1}(\bar{r})=\int_{4 \pi} Q(\bar{r}, \hat{s}) \hat{s} d \omega
$$

Where there are no sources, or where the sources are isotropic, $Q_{1}$ vanishes from Eq. (18). Then the vector irradiance is the gradient of the scalar fluence. This equation gives precision to the intuitive notion that there is net energy flow (i.e. non-zero vector irradiance) from regions of high energy density (high fluence) to regions of low energy density. By integrating the radiative transport equation over all directions $\omega$ at a point $r$, it yields

$$
\bar{\nabla} \cdot \bar{E}_{d}(\bar{r})=\mu_{d} \phi_{d}(\bar{r})+Q_{0}(\bar{r})
$$

where $Q_{0}$ is the zero-order source term of the volumetric source $Q$ which may be equated to [32]:

$$
Q_{0}(\bar{r})=\int_{4 \pi} Q(\bar{r}, \hat{s}) d \omega=\mu_{s}^{\prime}\left(1-r_{s}\right) \pi F_{0}(\bar{r}) e^{\mu_{t}^{\prime} z / \mu_{0}}
$$

Thus, regarding Eqs. (11), (12) and (19), we see that Eq. (18) states the net change in the diffuse radiant flux equals the intensity lost through absorption of the diffuse radiant fluence plus that gained through the scattering of collimated light into the diffuse portion of the radiance. Finally, substituting Eq. (16) into Eq. (18), we arrive at the classic diffusion equation:

$$
\nabla^{2} \phi_{d}(\bar{r})=3 \mu_{t}^{\prime} \mu_{a} \phi_{d}(\bar{r})-3 \mu_{t}^{\prime} Q_{0}(\bar{r})+3 \bar{\nabla} \cdot \bar{Q}_{1}(\bar{r})
$$

The diffusion equation has a simple solution in the case of a single isotropic point light source in an infinite medium

$$
\phi_{d}(\bar{r})=\frac{3 \mu_{t}^{\prime}}{4 \pi} \Phi \frac{e^{\mu_{e f f} r}}{r}
$$

where $\Phi$ is the power of the point light source, $r$ is the distance to the location of the point source, and $\mu_{e f f}$ is the effective attenuation or transport coefficient:

$$
\mu_{e f f}=\sqrt{3 \mu_{a} \mu_{t}^{\prime}}
$$

Correspondingly, the effective mean free path or penetration depth, $l_{e} f f$, can be defined by the reciprocal of the effective transport coefficient:

$$
l_{e f f}=\frac{1}{\mu_{e f f}}
$$

In the case of a single isotropic-point light source in an infinite medium, the point source results in an energy density in the volume with an exponential falloff. In the case of a scattering medium in a finite region of space, the diffusion equation must be solved subject to the appropriate boundary conditions. For different boundary conditions (index matching or mismatching, no incident diffuse light or diffuse light incident), Prahl accomplished the diffuse reflection $(T)$ and transmission $(R)$ of a slab with finite thickness by modeling the phase function as a delta-Eddington phase function [32].

The solutions indicate that, by measuring the diffuse reflection, collimated transmission $\left(T_{c}\right)$, and total transmission $\left(T_{t}=T+T_{c}\right)$ enable the determination of three optical properties: absorption coefficient $\left(\mu_{s}\right)$, scattering coefficient $\left(\mu_{s}\right)$ and anisotropy coefficient $(g)$. However, if only the diffuse reflection and total transmission measurements are available, only absorption and reduced scattering $\left(\mu_{s}^{\prime}=(1-g) \mu_{s}\right)$ coefficients can be calculated. Thus, in these cases, additional measurements for determining the scattering anisotropy factor are required.

\section{MATERIALS AND METHODS}

\subsection{Sample preparation}

Two different types of dental-resin composites (nano-filled and hybrid) with similar polymeric matrices (dimethacrylates: bisphenol A diglycidylether methacrylate [Bis-GMA], bisphenol A polyethylene glycol diether dimethacrylate [Bis-EMA], urethane dimethacrylate [UDMA], and triethylene glycol dimethacrylate [TEGDMA]), were studied. The characteristics of each dental-resin composite, according to the manufacturers, are shown in Table 1. Each specimen was prepared on a glass plate (Knittel GLASER, Bielefeld, Germany) with a hole bored using a high-speed hand drill with a round bur. After the placement of the composite, a clear plastic sheet (Acrylite Plus Clear, Tap Plastics, Dublin, CA, USA) was laid on the top and bottom of the mould and another glass plate was pressed onto the top to standardize the specimen thickness. Each specimen underwent photo-polymerization for 40 seconds using a light-curing unit (Bluephase, Ivoclar Vivadent AG, Liechtenstein) with an irradiance of $1100 \mathrm{~mW} / \mathrm{cm}^{2} \pm 10 \%$. After photo-polymerization, all glass plates were removed. Specimens were handled according to manufacturer's instructions. All specimens were prepared by the same user in order to minimize variability.

To analyze zirconia ceramic dental material, we used sintered LAVA $^{\mathrm{TM}}$ Zirconia provided by the manufacturer. The sintered ceramic were made using computer-aided design and manufacturing (CAD/CAM) procedures from pre-sintered zirconia blanks, the size of which had been increased to compensate for shrinkage during sintering in a special high-temperature furnace.

\subsection{The inverse-adding-doubling method}

The inverse-adding-doubling (IAD) method is a way to determine the optical properties of turbid media based on the adding-doubling approximation: inverse denotes a reversal of the usual process of calculating reflection and transmission from optical properties, and adding-doubling indicates the method used to resolve the radiative-transport equation. 


\begin{tabular}{|l|l|l|l|}
\hline Dental-Resin Composite & Organic Matrix & Inorganic Filler & Type \\
\hline Filtek Supreme XT & $\begin{array}{l}\text { Bis-GMA, Bis-EMA, UDMA, } \\
\text { TEGDMA }\end{array}$ & $\begin{array}{l}\text { Silica agglomerate, highly } \\
\text { dispersed silica }\end{array}$ & Nano-composite \\
\hline Z250 & Bis-GMA, Bis-EMA, UDMA & Silica, zirconia & Hybrid \\
\hline
\end{tabular}

TABLE 1 Characteristics of the two different types of dental-resin composites analyzed, according to the manufacturers

The adding-doubling method assumes knowledge of the reflection and transmission properties for a single thin homogeneous layer. For a thick sample, the reflection and transmission are estimated by repeated doubling until the desired thickness is reached. The radiance at the two surfaces of a thin slab can be attained if the phase function is known, since the multiple scattering is negligible. If an identical slab is added, the successive scattering back and forth between the component layers may be considered in order to calculate the radiance of the thicker slab. By iteratively adding other thin layers or doubling the total thickness, computation can be made for thicker slabs. With this quick and simple method, anisotropic scattering, internal reflecting, and arbitrary thick-layered media may be modeled [34].

As commented above, optical properties are used as input in order to calculate the reflectance and transmittance by means of the adding-doubling method. Nevertheless, in practice, total reflectance and transmittance values are measured, whereas the optical properties are unknown. Since the situation is the opposite, inverse estimation is needed. The IAD method, therefore, uses total reflectance and total transmittance values as inputs to estimate the values of the optical properties, i.e. absorption and reduced scattering coefficient. In the reverse approach, the optical properties of a sample can be determined by changing them at each iteration until the estimated reflectance and transmittance values match with the measured ones.

To solve the radiative transport equation, the iad program must be supplied with the experimental values of total diffuse reflectance $\left(M_{R}\right)$ and transmittance $\left(M_{T}\right)$ together with the values of the scattering anisotropy factor $(g)$ and the refraction index $(n)$ of the sample. The program guesses a set of optical properties $\left(\mu_{a}\right.$ and $\left.\mu_{s}^{\prime}\right)$ and then calculates values for reflectance $\left(M_{R}\right)$ and transmittance $\left(M_{T}\right)$. This process is repeated until the calculated and measured values of reflectance $\left(M_{R}\right)$ and transmittance $\left(M_{T}\right)$ are within a specified tolerance (for the sum of both relative differences, the tolerance default value is $0.01 \%$ ).

In addition, particle simulations based on random sampling from appropriate probability distributions are also solutions for the radiative transfer equation $[35,36]$. This particle simulation represents the most common Monte Carlo application in radiative transfer.

The Monte Carlo simulation is a statistical method that calculates the trajectories of a great number of photons and, as a result, presents the reflectance and transmission of a sample for a given set of optical parameters. The use of this simulation minimizes systematic errors, considers the scattering phase function, and also takes into account the measuring ge- ometry. A limiting consideration is that the accuracy of quantities calculated increases only with the square root of the number of photon histories, making the Monte Carlo particle simulation a computationally costly method. Nevertheless, as computing power is progressively becoming cheaper, this technique is being more widely applied, for example, in tissue optics $[37,38]$. The inverse Monte Carlo simulation was included in the iad program to achieve an accurate evaluation of the sample's optical properties. The measurements of the total diffuse reflectance $\left(M_{R}\right)$ and transmittance $\left(M_{T}\right)$ used in the IAD method to determine the optical absorption coefficient $\left(\mu_{a}\right)$ and reduced scattering coefficient $\left(\mu_{s}^{\prime}\right)$ are modeled by the Monte Carlo simulation technique, which uses a stochastic simulation of light interaction with biological media [39]-[41].

Figure 1 shows a schematic diagram of the iad-program logic $[32,40]$. At the beginning, lost light through the sample edges is fixed at zero and the program calculates a coarse grid for measured reflectance and transmittance values with stipulated absorption coefficient, reduced scattering coefficient, and scattering anisotropy factor (in the case of available $g$ values, they can be assigned by means of a command line of the program which switches this variable to a fixed value). The diffuse and direct reflectance and transmittance are calculated by adding-doubling algorithm for a set of optical properties $\left(\mu_{a}, \mu_{s}^{\prime}\right.$ and $\left.g\right)$. Then, the lost light for the diffuse and direct fractions is proportionally subtracted and the total reflectance and transmittance is calculated. The loop is repeated by changing the optical properties until the calculated and the measured values match each other. When this occurs, the fraction of lost light is calculated by performing the Monte Carlo simulation. With the lost-light correction, new optical coefficients are sought and the process is repeated again until the changes in the values of the absorption and scattering coefficients are less than $0.1 \%$. In this work, using the iad program [15], the absorption coefficient $\left(\mu_{a}\right)$ and reduced scattering coefficient $\left(\mu_{s}^{\prime}\right)$ were attained from the experimental values of total diffuse reflectance $\left(M_{R}\right)$ and transmittance $\left(M_{T}\right)$ along with those of the scattering anisotropy factor $(g)$ calculated in previous works [27]. The experimental values of total diffuse reflectance $\left(M_{R}\right)$ and transmittance $\left(M_{T}\right)$ were determined by measuring total reflection and transmission with an integrating-sphere setup.

For the measurement of the reflected and transmitted light, the sample is situated, respectively, at the exit and entrance port of a sphere. Normally the detector is placed on the inner sphere surface. The integrating-sphere theory accounts for the losses caused by absorption from the sphere wall and light escaping the sphere. The power detected depends on the total light remaining within the sphere and on the relative (to the total sphere area) size of the detector $[42,43]$. Together with 


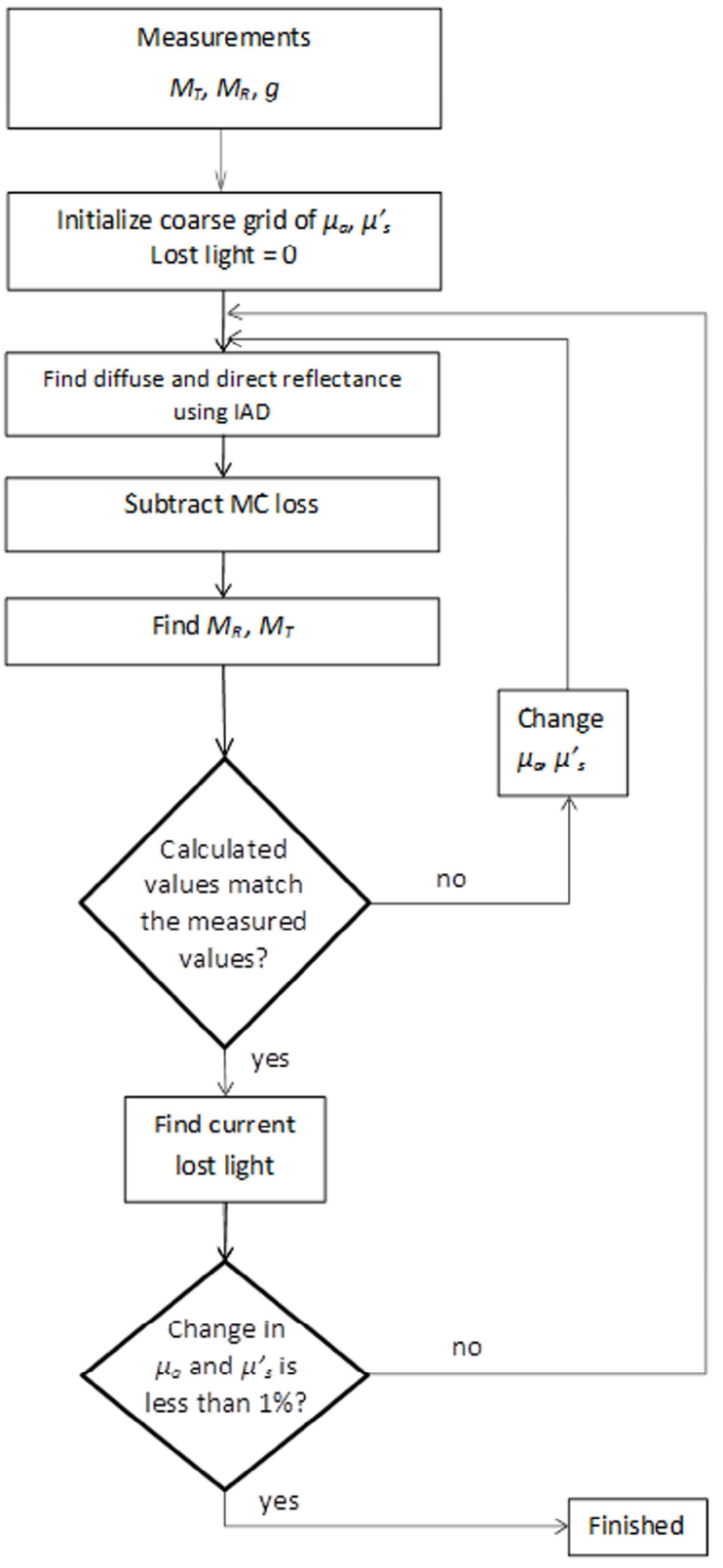

FIG. 1 Schematic diagram of the iad-program logic with diffuse reflectance $\left(M_{R}\right)$ and transmittance $\left(M_{T}\right)$ along with scattering anisotropy factor $(g)$ experimental values as input parameters.

the reflectance and transmittance values, a critical input of the iad program is the integrating-sphere wall reflectance $r_{w}$. Two measurements (Figure 2) are needed to experimentally determine $r_{w}$ by means of the expression

$$
\frac{1}{r_{w}}=a_{w}+a_{d} r_{d}\left(1-a_{e}\right)+a_{s} r_{s t d}\left(1-a_{e}\right) \frac{r_{\text {std }}^{\text {diffuse }}}{r_{\text {std }}^{\text {diffuse }}-r_{0}^{\text {diffuse }}}
$$

where $a_{w}, a_{d}, a_{e}$, and $a_{s}$ are, respectively, the sphere wall (ports excluded), the detector, the entrance port, and the sample, relative areas to the total sphere wall area; $r_{d}$ and $r_{s t d}$ are, respectively, the reflectance of the detector and the reference standard.

Eq. (24) illustrates the difficulty in determining accurate values of the sphere-wall reflectance from the measurements. The two diffuse reflectances $r_{\text {std }}^{\text {diffuse }}$ and $r_{0}^{\text {diffuse }}$ differ only in the amount of diffuse light leaking from the sphere when the port
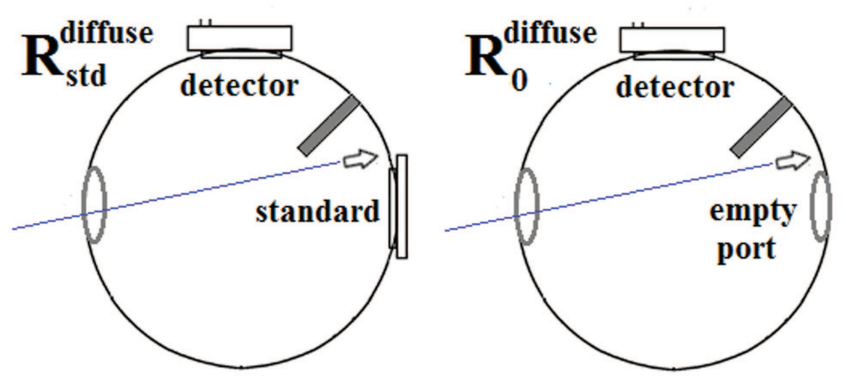

FIG. 2 Measurements of diffuse reflectance needed to determine the integrating-sphere wall reflectance $\left(r_{w}\right)$.
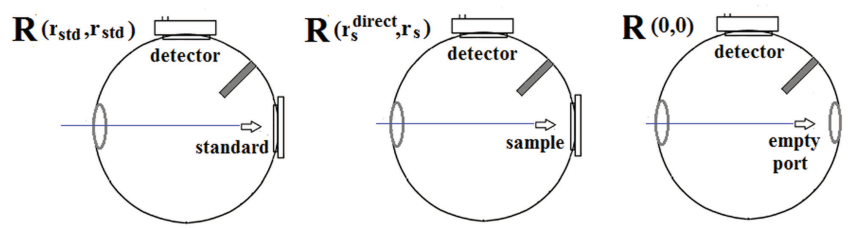

FIG. 3 Single-integrating sphere measurements of reflection needed to extract the total diffuse reflectance of the sample $M_{R}$.

is empty. Consequently, the difference will be small (roughly proportional to the relative area of the sample port) and any errors in the measurements will be magnified when the division is performed. For a single-integrating-sphere setup using collimated light (i.e. first incident on the sample [Figure 3]), the total diffuse reflectance $M_{R}$ is defined in terms of easily measurable sample and standard reflection as

$$
M_{R}=r_{s t d} \frac{R\left(r_{s}^{\text {direct }}, r_{s}\right)-R(0,0)}{R\left(r_{s t d}, r_{s t d}\right)-R(0,0)}
$$

where $R(0,0)$ is subtracted to discount the effect of the incident light that hits the sphere wall before the sample, which is not uniformly diffuse (the light must hit the walls one more time before becoming uniform). Under the same conditions (single-integrating-sphere setup using collimated light [Figure 4]), the total diffuse transmittance $M_{T}$ is defined in terms of sample reflection and incident light measurements as

$$
M_{T}=\frac{T\left(t_{s}^{\text {direct }}, t_{s}\right)-T_{\text {dark }}}{T(0,0)-T_{\text {dark }}}
$$

where $T_{\text {dark }}$ constitutes the background signal detected when obscuring the source. Note that, although it is necessary to subtract this signal from every measurement, this subtraction is canceled in Eq. (25).

\subsection{Experimental setup}

As commented above, it is difficult to achieve accurate values for the integrating-sphere wall reflectance $r_{w}$, since the quantity of interest is a small difference between two measurements. In this work, this was done under repeatability conditions of measurement in order to determine the corresponding uncertainty: 20 measurements of the two diffuse reflectances were taken, from which 20 values of the spherewall reflectance were determined. Then, we calculated the average of the 20 different values and its corresponding typeA uncertainty, expressed as a standard deviation [44]. In ad- 


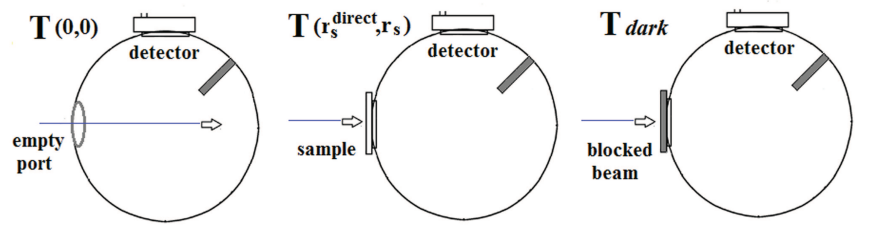

FIG. 4 Single-integrating sphere measurements of transmission needed to extract the total diffuse transmittance of the sample $M_{T}$.

dition, to minimize the uncertainty, we repeated this procedure three times, resulting in three sphere-wall reflectance values. Finally, the sphere-wall reflectance was considered to be the average of this three values while its associated uncertainty was computed considering the law of propagation of uncertainties [44]. In Eq. (24), the reflectance of the detector, $r_{d}$, was assumed to be negligible. In all the calculations, the manufacturer-specified values for $r_{s t d}$ were considered.

Reflectance and transmittance values were determined from the direct measurements of total reflection and transmission. It should be noted that reflectance and transmittance are relative quantities which are independent of the intensity of the incident light.

For each dental biomaterial studied, the reflectance and transmittance values were joined with the corresponding scattering anisotropy value for computing the absorption and the reduced scattering coefficients by means of the IAD method. Again, this was done under repeatability conditions of measurement: the metrological procedure was repeated 20 times with each sample in order to determine the uncertainty associated to the absorption and the reduced scattering coefficients. From these 20 measurements, we calculated the average value of both coefficients and their corresponding type-A uncertainties, expressed as standard deviations [44].

From the average values of the absorption and the reduced scattering coefficients, the effective transport coefficient, $\mu_{e f f}$, and the effective mean free path, $l_{e f f}$, were calculated for each biomaterial. The uncertainties associated to these optical properties were computed considering the law of propagation of uncertainties [44], taking into account the uncertainties of the corresponding absorption and reduced scattering coefficients.

It is noteworthy that, in the present work, spectral variations not only of the refraction index but also of the scattering anisotropy factor were taken into account. Despite their potential repercussion in accurateness, these aspects are commonly neglected when using the IAD method for determining the optical properties of biological media. Sometimes a single value in the entire spectral range investigated is assumed for $n$ and/or $g$, but it is not particularly associated with the medium under study [16, 45]; other times, $g$ measurements are performed specifically for the medium but with a single wavelength [46]-[48].

As demonstrated elsewhere [27]-[29], scattering anisotropy factor values of the dental biomaterials are farther away from zero (isotropy) and also change with the wavelength. This is

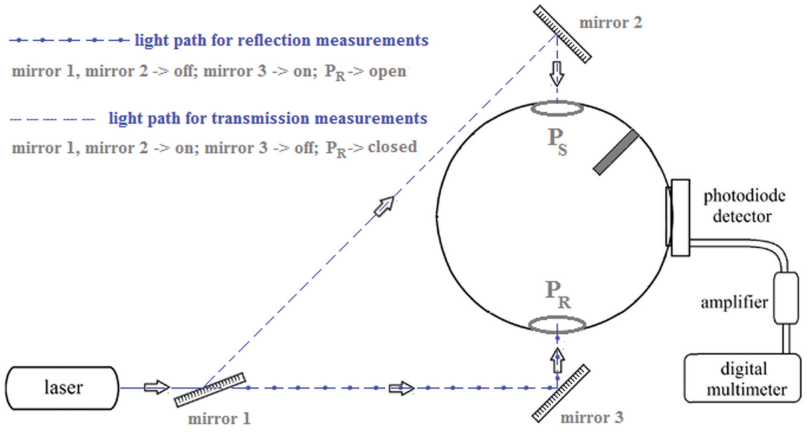

FIG. 5 Experimental setup used in reflection and transmission measurements on the dental biomaterials. $P_{S}$ is the sample port and $P_{R}$ is the reflection entrance port (stopped with a port plug for transmission measurements). A folding mount allows mirror 1 to be lifted away so that the beam reaches mirror 3 for reflection measurements.

expected to affect the spectral values of other g-related optical properties. On the other hand, the refractive index of the material is important in determining the optical properties because it affects the reflection at the border lines caused by sample surface and filler particles or matrix, in the case of the dental-resin composites $[49,50]$. Particularly, for the dentalresin composites, since they are composed of inorganic filler particles immersed in an organic matrix, changes in the refractive index inside the material may have a substantial influence. Since data provided by the manufacturers is restricted to separated values of filler and matrix refractive indexes, we measured the overall refractive index of each dental-resin composite samples with an Abbe refractometer (PCE Iberica, Spain). For the zirconia ceramic, we considered the refractive index values given elsewhere [51].

Below, we briefly describe the laser-integrating-sphere-based setup used to undertake the reflection and transmission measurements on the dental biomaterial samples. A simplified diagram of the experimental setup used is shown in Figure 5. Basically, it consists of a laser source, a mirror assembly, a three-port integrating sphere (Oriel 70674, Newport, USA), and a silicon photodiode detector (S2281, Hamamatsu, Japan) connected to a measuring system. The measuring system was composed by a photosensor amplifier (C2719 Hamamatsu, Japan), a multimeter (34401A, Agilent Technologies, USA), and a personal computer. Throughout the measuring procedure, the photodiode was attached to the same port of the sphere. This port was perpendicular to the sample port and a baffle was located between the two ports to prevent the detection of specular reflected light. Depending on the stage of the measuring procedure, by actuating on the mirror assembly, the laser beam was aimed at the corresponding entrance port: the sample port, for the transmission measurements; and the opposite port, for the reflection measurements. It bears noting that, with this configuration, the sample port was fixed and the entrance port was variable (as opposed to traditional configurations). In this way, the movement of the sample between reflection and transmission measurements was avoided. For the reflection measurement, mirrors 1 and 2 were plugged, while mirror 3 was put into action in order to aim the laser beam to the reflection entrance port, $P_{R}$. The mirrors of the assembly were interchanged for the transmission measure- 
ments: 3 was sealed off while 1 and 2 actuated to direct the beam to the transmission entrance port, i.e. the sample port, $P_{S}$. At this stage of the measuring procedure, $P_{R}$ was obturated with a specific port plug of the same material as the inner sphere wall (barium sulfate). For the sphere-wall reflectance measurements, with the mirrors of the assembly set as in the reflection measurements, the integrating sphere was rotated so that the laser beam first irradiated the sidewall of the sphere between the baffle and the sample port.

With the aim of calculating the optical properties of the biomaterials at different wavelengths, measurements were made using two laser sources: a He-Ne laser source with a power of $12.0 \mathrm{~mW}$ (30564, Research Electro Optics, USA), which provided a wavelength of $632.8 \mathrm{~nm}$; and a tunable ion-argon laser source with $1000 \mathrm{~mW}$ of maximum total power (Stellar-Pro-L ML/1000, Modu-Laser, USA), which provided wavelengths of $457.9,488.0$, and $514.5 \mathrm{~nm}$. For each of these wavelengths, the whole process of achieving the sphere-wall reflectance and the samples' reflectance and transmittance was performed.

Before the processing, all the data were corrected to reduce effects from background noise, due to laboratory lighting and photodiode dark current (response in the absence of input light-signal, i.e. with the detector in the dark). The background noise was corrected by subtracting from the measured signal values the background signal detected when obscuring the laser source.

The experimental procedure employed fulfilled all the necessary requirements to provide optical-property values with lower associated uncertainties. Firstly, collimated rather than diffuse irradiance was used. Diffuse light may irradiate the sample through the beam by first irradiating the sidewall of the sphere. Alternatively, collimated light may directly irradiate the sample, as occurred in our setup. In the diffuse case, there is a signal registered by the detector due to the light within the sphere, even when no sample is present. Thus all measurements are made with a high background signal. Noise in this background signal, especially with lowreflectance samples, result in uncertainties in the calculation of the reflectance [13]. In the collimated case the sample reflectance acts as the source, and the background signal is due only to the laboratory ambient lighting (almost inappreciable, since it must enter the sphere before reaching the detector) and to the photodiode dark current. Thus, noise in this background signal is expected to be lower with the collimated irradiance that we used. In addition, before processing, we corrected all the data to reduce the effects of this background noise.

Secondly, major difficulties arise in making precise collimated transmission measurements for samples with greater extinction or transport coefficients, due to the low signal registered by the detector. Although the use of a photomultiplier rather than a photodiode could be expected to improve the measurement, this constitutes a much more expensive option. Without the collimated transmission measurement the reduced scattering coefficient cannot be separated into the scattering coefficient and the scattering anisotropy factor $g$, except if the value of the former one $(g)$ is already known. In previous works
[27]-[29], goniometric measurements were made in order to recover the experimental phase functions and the corresponding $g$ values of dental-resin composites and zirconia ceramic. Given the low uncertainties found, the use of these $g$ values offered greater guarantees.

On other hand, with a double-integrating-sphere system, in the reflectance sphere an additional uncertainty will be caused by the signal returning from the transmittance sphere through the sample. For collimated irradiance this signal is not directly proportional to the reflectance of the sample (but rather the transmittance) and is maximal when there is no absorption. This leads to a greater uncertainty in reflectance than with a single-integrating-sphere system [13]. Thus, with the use of a single-sphere system the uncertainty in reflectance decreased because of the exchange of light between the spheres was avoided. Finally, with conventional integrating-sphere configurations, the reflectance and transmittance measurements were sequentially undertaken keeping the entrance port and changing the sample position. By means of a mirror assembly, this disadvantage was overcome and, consequently, also the uncertainty involved was expected to be reduced. It should be pointed out that, since we did not seek to measure changes in optical properties of biomaterials with external stimulus (such as heating o light-curing), simultaneous measurement of reflection and transmission were not required in this work.

In summary, the experimental procedure employed offered the advantage of using single-sphere system (avoiding light interactions between the spheres) without their disadvantage, since the movement of the sample between reflection and transmission measurements was surpassed with the incorporation of an adequate mirror assembly. This setup is appropriate for steady-state (time independent) biomaterial studies and also recommended when low uncertainties are sought, e.g. in comparative analyses of different materials.

\subsection{Input parameters for the iad program}

\begin{tabular}{|c|c|}
\hline Material & Thickness $(\mathrm{mm})$ \\
\hline Nanocomposite & 0.41 \\
\hline Hybrid composite & 0.80 \\
\hline Zirconia ceramic & 0.3 \\
\hline
\end{tabular}

TABLE 2 Thicknesses of the samples used in the measurements (values determined with $0.01 \mathrm{~mm}$ in sensitivity).

As indicated above, for each wavelength analyzed, the absorption coefficient $\left(\mu_{a}\right)$ and reduced scattering coefficient $\left(\mu_{s}^{\prime}\right)$ were determined from the experimental values of total diffuse reflectance $\left(M_{R}\right)$, transmittance $\left(M_{T}\right)$, and scattering anisotropy factor $(g)$ using the iad program. Together with this data, the program requires some additional parameters related not only to the samples but also to the experimental setup used for the measurements.

In addition to $M_{R}, M_{T}$ and $g$ spectral values, the parameters related to the samples required are the thickness and the refractive index values. Table 2 shows the thicknesses of the sample determined using a Series 500, Mitutoyo digital caliper (0.01 in sensitivity). The spectral values of the refractive in- 
dex taken into account are listed in Table 3. In the latest ta-

\begin{tabular}{|l|l|l|l|}
\hline$\lambda(\mathrm{nm})$ & $\begin{array}{l}\text { Nano- } \\
\text { composite }\end{array}$ & $\begin{array}{l}\text { Hybrid } \\
\text { composite }\end{array}$ & $\begin{array}{l}\text { Zirconia } \\
\text { ceramic }\end{array}$ \\
\hline 457.9 & 1.5390 & 1.5320 & 2.19337 \\
\hline 488.0 & 1.5385 & 1.5315 & 2.18254 \\
\hline 514.5 & 1.5380 & 1.5315 & 2.17471 \\
\hline 632.8 & 1.5380 & 1.5315 & 2.15169 \\
\hline
\end{tabular}

TABLE 3 Spectral refraction index values considered for the dental biomaterials analyzed.

ble, it can be seen that the variation of the refractive index with the wavelength is more important for the zirconia ceramic than for the two dental-resin composites. As pointed out previously, for the composites, the values of the refractive index were measured with a commercial device. On the other hand, the values of the refractive index taken into account for the zirconia ceramics were theoretical values provided elsewhere [51]. This may explain the spectral variation differences in the refractive indexes considered, along with the differences in nature of the materials.

As noted above, among the setup parameters required for the program to describe the particular experiment performed, the integrating-sphere wall reflectance $r_{w}$ is critical. Table 4 lists the experimental $r_{w}$ values determined as indicate above, together with their corresponding uncertainties.

Additional input parameters mandatory for executing the iad program are the diameters of: the laser beam $(2 \mathrm{~mm})$, the integrating sphere $(158.2 \mathrm{~mm})$ and the sphere ports $(15 \mathrm{~mm}$, in the entrance and sample ones; $11 \mathrm{~mm}$, in the detector one).

\section{RESULTS AND DISCUSSION}

For each dental biomaterial analyzed, the spectral values of the optical properties determined are presented in Tables 5 to 7 with their associated uncertainties. These tables show the experimental values of: the absorption coefficient, $\mu_{a}$; the reduced scattering coefficient, $\mu_{s}^{\prime}$; the scattering anisotropy factor, $g$; the scattering coefficient, $\mu_{s}$; the effective transport coefficient, $\mu_{e f f}$; and the effective mean free path, $l_{e f f}$.

As pointed out above, the values of $\mu_{a}$, and $\mu_{s}^{\prime}$ were determined from the measurements, and $g$ values were calculated in previous works [27]. By applying Eq. (7), $\mu_{s}$ values were calculated from the values of $\mu_{s}^{\prime}$ and $g$. Finally, $\mu_{e f f}$ and $l_{e f f}$ values were calculated as indicated in Eq. (22) and (23), respectively.

Data in Tables 5 to 7 confirm the suitability of diffusion theory for the dental biomaterials studied, since the values of the reduced scattering coefficient are much higher than those of the absorption coefficient. On the other hand, the data show that the spectral variations of the reduced scattering coefficient, $\mu_{s}^{\prime}$, did not agree with those of the scattering coefficient, $\mu_{s}$, for all the biomaterials studied. For both dental-resin composites $\mu_{s}^{\prime}$ decreased monotonically in the wavelength range whereas $\mu_{s}$ presented a minimum. However, for the zirconia ceramic, both scattering coefficients showed similar spectral behavior, with higher values for the reduced one. This illustrates the expected influence of the scattering anisotropy factor values. The factor of $1-g$ varied between $1.192 \pm 0.003$ and $1.247 \pm 0.005$ for the zirconia ceramic, while this factor varied from $0.01700 \pm 0.00021$ to $0.0513 \pm 0.0004$ for the nanocomposite, and from 0.04570 \pm 0.00014 to $0.1445 \pm 0.0003$ for the hybrid composite. Zirconia $g$ values are considerably closer to the isotropy $(g=0)$ than the composites $g$ values, which are strongly forward directed (near to 1). Thus, scattering anisotropy has a markedly distinct impact on the optical properties of zirconia ceramics and dental-resin composites, as demonstrated. For comparisons among the different dental biomaterials at the wavelengths evaluated, the values of the absorption coefficient, $\mu_{a}$, the scattering coefficient, $\mu_{s}$, the effective transport coefficient, $\mu_{e f f}$, and the effective mean free path, $l_{\text {eff }}$, are jointly represented in Figures 6 to 9, respectively. In addition, to help the evaluation of the differences between the two dental-resin composites, Figure 10 displays simultaneously their values of the reduced scattering coefficient, $\mu_{s}^{\prime}$. For the zirconia ceramic, $\mu_{s}^{\prime}$ spectral values are represented in Figure 11. The inclusion of $\mu_{s}^{\prime}$ values in this figure assists a clearer confirmation of the scattering anisotropy effects.

As can be seen, differences in the optical properties of the three dental biomaterials studied are significant. By virtue of the low uncertainties found, the experimental values calculated could be distinguished and compared. The absorption coefficient had similar spectral variations for both dental-resin composites, but completely different for the zirconia ceramic. In the case of the scattering coefficient, the spectral values appeared to follow more parallel trends for the three biomaterials, with higher values for the nanocomposite. Comparisons of the reduced scattering coefficients of only the dental resins revealed that the highest values were presented by the hybrid composite except at the 632.8-nm wavelength. The scattering anisotropy distinct impacts on the optical properties of zirconia ceramic and on those of the dental-resin composites can be clearly checked observing Figures 7, 10, and 11.

For both dental-resins, the effective transport coefficient showed similar spectral trends. On the contrary, the effective transport coefficient of the zirconia ceramic presented not only higher values but also a different spectral tendency compared with the composites. The effective mean free path seemed to present rather dissimilar spectral trends for each dental biomaterial studied, with a marked increment at $632.8 \mathrm{~nm}$ in the case of the hybrid composite.

These data may be valuable for clinical applications, since the effective mean path or penetration depth is a measure of how deeply laser light can penetrate into the material. Furthermore, with the knowledge of $\mu_{a}, \mu_{s}$ and $g$, we can estimate spectral values and color coordinates from simulated reflectances without the need of preparing samples for additional measurements [52]-[54]. 


\begin{tabular}{|c|c|c|c|c|}
\hline$\lambda(\mathrm{nm})$ & 457.9 & 488.0 & 514.5 & 632.8 \\
\hline$r_{w}$ & $0.9805 \pm 0.0009$ & $0.98263 \pm 0.00025$ & $0.9769 \pm 0.0006$ & $0.98269 \pm 0.00016$ \\
\hline
\end{tabular}

TABLE 4 Reflectance values of the integrating-sphere wall, with their associated uncertainties.

\begin{tabular}{|c|c|c|c|c|c|c|}
\hline$\lambda(\mathrm{nm})$ & $\mu_{a}\left(\mathrm{~mm}^{-1}\right)$ & $\mu_{s}^{\prime}\left(\mathrm{mm}^{-1}\right)$ & $g$ & $\mu_{s}\left(\mathrm{~mm}^{-1}\right)$ & $\mu_{\text {eff }}\left(\mathrm{mm}^{-1}\right)$ & $l_{\text {eff }}(\mathrm{mm})$ \\
\hline 457.9 & $0.0704 \pm 0.0014$ & $0.999 \pm 0.005$ & $0.9617 \pm 0.0003$ & $26.08 \pm 0.25$ & $0.475 \pm 0.005$ & $2.104 \pm 0.021$ \\
\hline 488.0 & $0.0350 \pm 0.0006$ & $0.851 \pm 0.012$ & $0.9487 \pm 0.0004$ & $16.6 \pm 0.3$ & $0.305 \pm 0.004$ & $3.28 \pm 0.04$ \\
\hline 514.5 & $0.0281 \pm 0.0017$ & $0.769 \pm 0.005$ & $0.9570 \pm 0.0004$ & $17.87 \pm 0.20$ & $0.259 \pm 0.008$ & $3.86 \pm 0.12$ \\
\hline 632.8 & $0.01705 \pm 0.00104$ & $0.7400 \pm 0.0039$ & $0.98297 \pm 0.00021$ & $43.5 \pm 0.6$ & $0.197 \pm 0.006$ & $5.08 \pm 0.16$ \\
\hline
\end{tabular}

TABLE 5 Optical properties with their associated uncertainties for the nanocomposite.

\begin{tabular}{|c|c|c|c|c|c|c|}
\hline$\lambda(\mathrm{nm})$ & $\mu_{a}\left(\mathrm{~mm}^{-1}\right)$ & $\mu_{s}^{\prime}\left(\mathrm{mm}^{-1}\right)$ & $g$ & $\mu_{s}\left(\mathrm{~mm}^{-1}\right)$ & $\mu_{\text {eff }}\left(\mathrm{mm}^{-1}\right)$ & $l_{\text {eff }}(\mathrm{mm})$ \\
\hline 457.9 & $0.0586 \pm 0.0005$ & $1.343 \pm 0.004$ & $0.8710 \pm 0.0003$ & $10.41 \pm 0.04$ & $0.4964 \pm 0.0021$ & $2.014 \pm 0.009$ \\
\hline 488.0 & $0.0382 \pm 0.0004$ & $1.191 \pm 0.006$ & $0.8555 \pm 0.0003$ & $8.24 \pm 0.04$ & $0.3753 \pm 0.0020$ & $2.664 \pm 0.014$ \\
\hline 514.5 & $0.0284 \pm 0.0012$ & $1.028 \pm 0.004$ & $0.8558 \pm 0.0003$ & $7.13 \pm 0.03$ & $0.300 \pm 0.006$ & $3.33 \pm .07$ \\
\hline 632.8 & $0.00221 \pm 0.00011$ & $0.7261 \pm 0.0014$ & $0.95429 \pm 0.00014$ & $15.89 \pm 0.06$ & $0.0695 \pm 0.0018$ & $14.4 \pm 0.4$ \\
\hline
\end{tabular}

TABLE 6 Optical properties with their associated uncertainties for the hybrid composite.

\begin{tabular}{|c|c|c|c|c|c|c|}
\hline$\lambda(\mathrm{nm})$ & $\mu_{a}\left(\mathrm{~mm}^{-1}\right)$ & $\mu_{s}^{\prime}\left(\mathrm{mm}^{-1}\right)$ & $g$ & $\mu_{s}\left(\mathrm{~mm}^{-1}\right)$ & $\mu_{\text {eff }}\left(\mathrm{mm}^{-1}\right)$ & $l_{\text {eff }}(\mathrm{mm})$ \\
\hline 457.9 & $0.0203 \pm 0.0005$ & $14.63 \pm 0.12$ & $-0.192 \pm 0.003$ & $12.27 \pm 0.10$ & $0.945 \pm 0.013$ & $1.058 \pm 0.015$ \\
\hline 488.0 & $0.02889 \pm 0.00025$ & $14.15 \pm 0.05$ & $-0.247 \pm 0.005$ & $11.35 \pm 0.06$ & $1.109 \pm 0.005$ & $0.902 \pm 0.004$ \\
\hline 514.5 & $0.0604 \pm 0.0007$ & $11.47 \pm 0.07$ & $-0.233 \pm 0.003$ & $9.30 \pm 0.06$ & $1.446 \pm 0.009$ & $0.692 \pm 0.005$ \\
\hline 632.8 & $0.00947 \pm 0.00008$ & $20.696 \pm 0.014$ & $-0.2074 \pm 0.0024$ & $17.14 \pm 0.04$ & $0.767 \pm 0.003$ & $1.304 \pm 0.005$ \\
\hline
\end{tabular}

TABLE 7 Optical properties with their associated uncertainties for the zirconia ceramic.

\section{CONSLUSION}

Restoration final success and optimal quality imply a deepest physical understanding of the optical properties in dental biomaterials. By means of the inverse-adding-doubling (IAD) method, the absorption coefficient, and the reduced scattering coefficient can be determined by transmittance and reflectance measurements. The additional measurement of the phase function performed by goniometry allows the separation of the reduced scattering coefficient into the scattering coefficient and the scattering anisotropy factor. In the present work, we used the IAD method to combine transmittance and reflectance measurements performed using the integrating sphere setup with the results of the previous goniometric measurements. We experimentally analyzed two different dental-resin composites (hybrid and nano-filled) and one type of zirconia ceramic. The experimental procedure was conducted under repeatability conditions of measurement in order to determine the uncertainty associated to the optical properties of the biomaterials. It is noteworthy that spectral variations not only of the refraction index but also of the scattering anisotropy factor were taken into account.

The setup used is appropriate for steady-state (time independent) biomaterial optics studies and presented the advantage of using single-sphere system (avoiding light interactions between the spheres) without their disadvantage, since the movement of the sample between reflection and transmission measurements was surpassed with the incorporation of an adequate mirror assembly.
The whole experimental procedure fulfilled all the necessary requirements to provide optical-property values with lower associated uncertainties. Thus, it is recommended for the comparative analysis of different materials.

For the dental biomaterials studied, the results agree with the application of the diffusion theory, since the reduced scattering coefficient had values much higher than the absorption coefficient. On the other hand, the data attained show a disparity between the spectral variations of the scattering coefficient and the reduced scattering coefficient for the dental resins but not for the zirconia ceramic, illustrating the influence of the scattering anisotropy value. These results demonstrated that the scattering anisotropy has clearly distinct impacts on the optical properties of zirconia ceramic and dental-resin composites, as expected, since zirconia $g$ values are much closer to the isotropy than the composites $g$ values, which are strongly forward directed.

It bears noting the low uncertainties found, which allowed the experimental values calculated to be distinguished and compared. The results reveal that the absorption coefficient had similar spectral variation for both dental-resin composites, but thoroughly different for the zirconia ceramic. In the case of the scattering coefficient, the spectral values appeared to follow more parallel trends for the three biomaterials, with higher values for the nanocomposite. Comparing the reduced scattering coefficients of only the dental-resins, we found that the highest values were presented by the hybrid composite except at the $632.8 \mathrm{~nm}$-wavelength. For both dental-resins, the effective transport coefficient showed similar spectral trends. On 

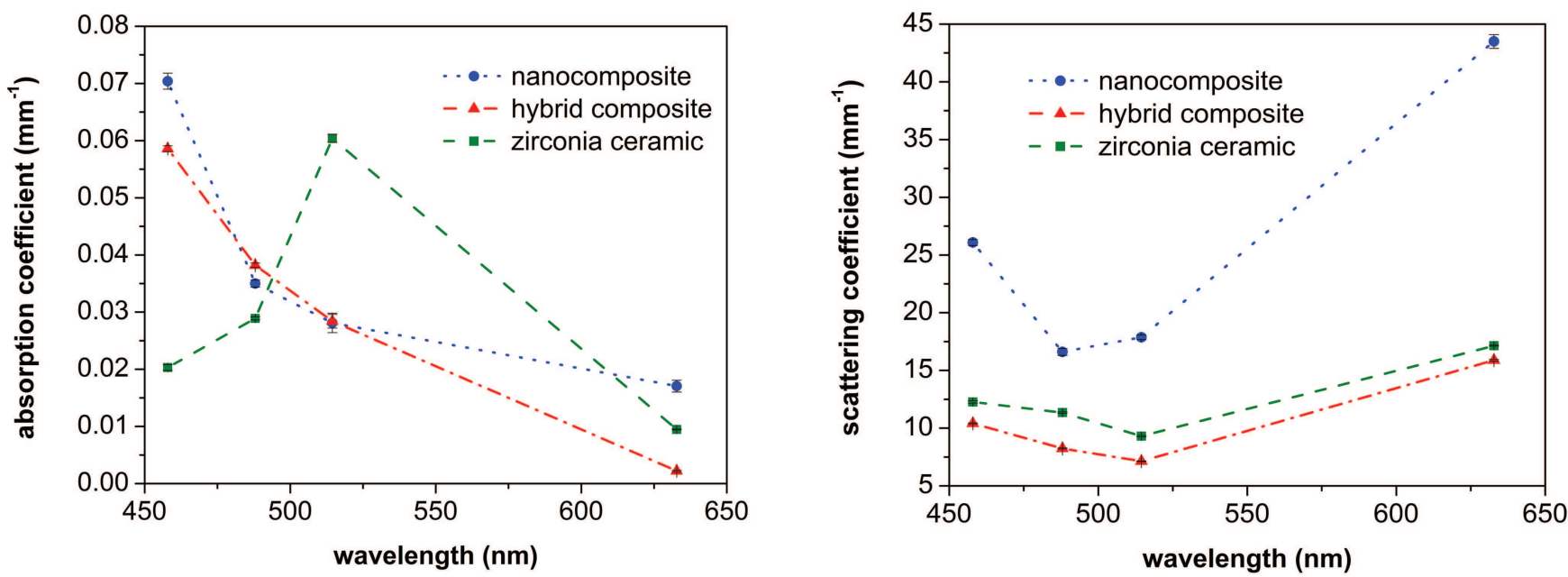

FIC. 6 Spectral values of the absorption coefficient for the dental biomaterials studied. FIG. 7 Spectral values of the scattering coefficient for the dental biomaterials studied.
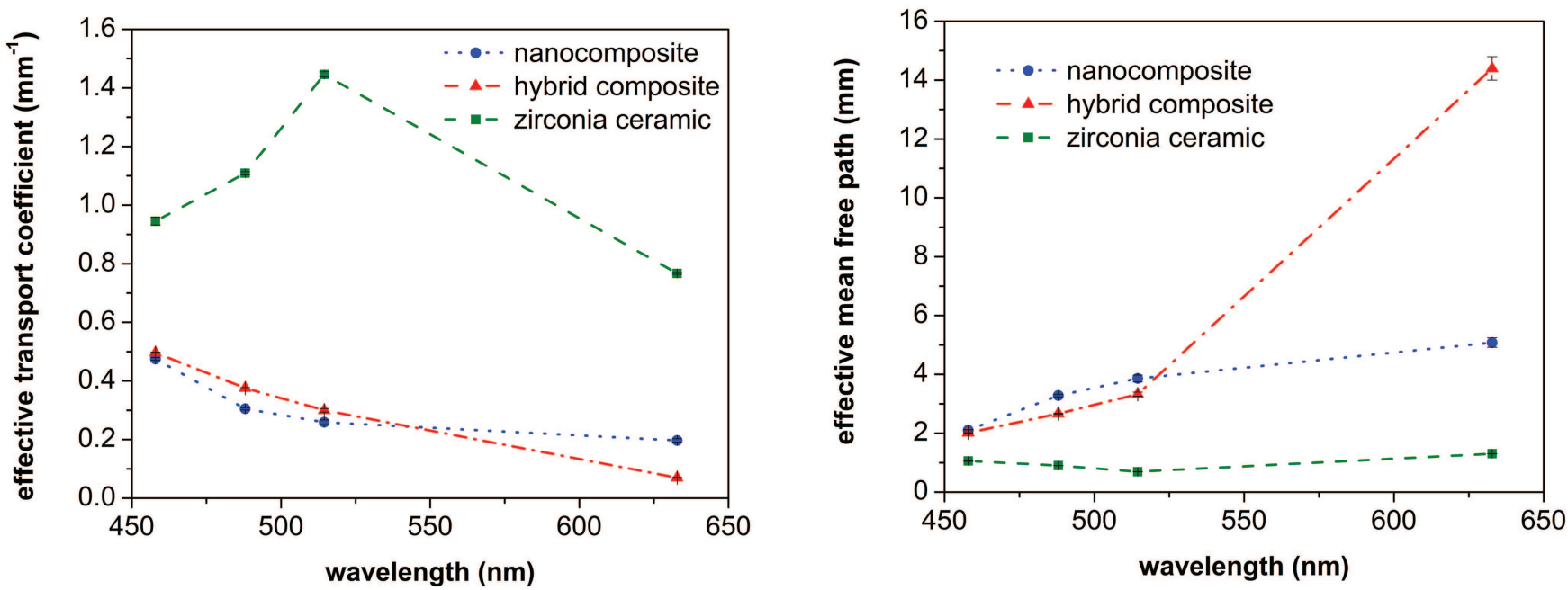

FIG. 8 Spectral values of the effective transport coefficient for the dental biomaterials FIG. 9 Spectral values of the effective mean free path for the dental biomaterials studied studied.
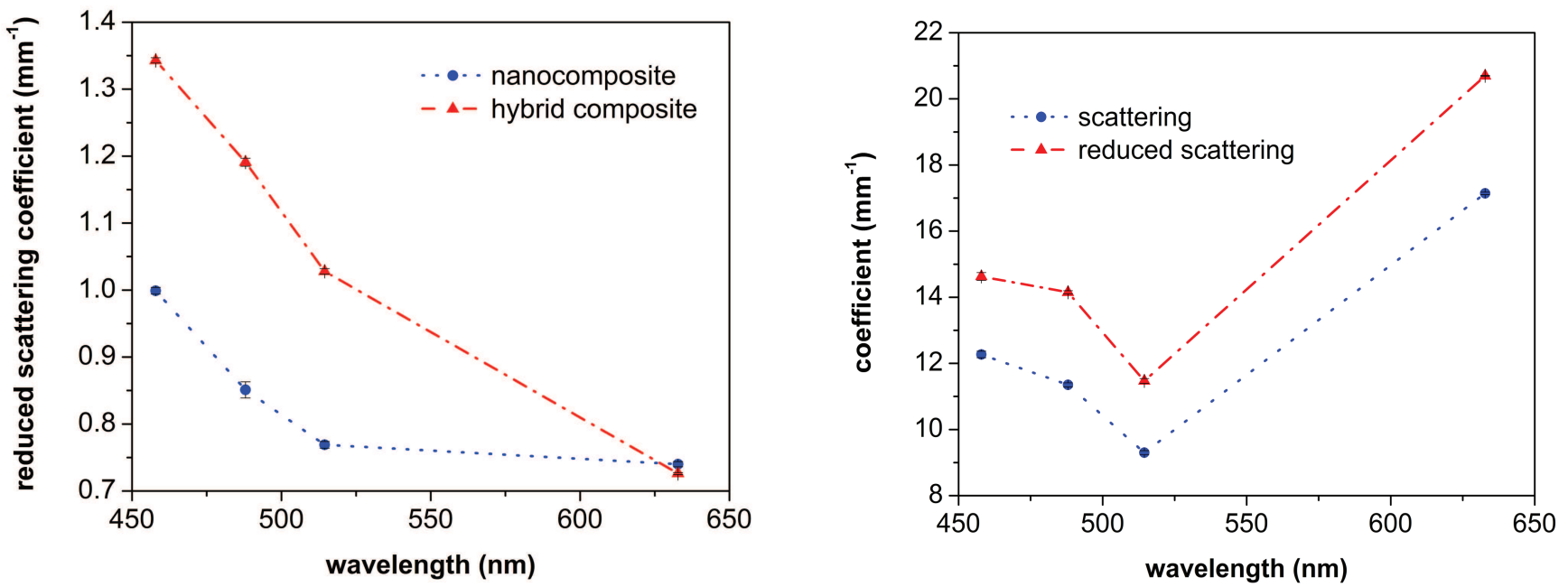

FIG. 10 Spectral values of the reduced scattering coefficient for the dental-resin compos- FIG. 11 Spectral values of the scattering coefficients for the zirconia ceramic studied. ites studied.

the contrary, the effective transport coefficient of the zirconia ceramic presented not only higher values but also a different spectral trend compared with the composites. The effective mean free path seemed to show somewhat dissimilar spectral trends for each dental biomaterial studied, with a marked increment at $632.8 \mathrm{~nm}$ in the case of the hybrid composite.
These data may be valuable for clinical applications, since the effective mean path or penetration depth is a measure of how deep laser light can penetrate the material. Additionally, with the knowledge of $\mu_{a}, \mu_{s}$, and $g$ spectral values, color coordinates may be estimated without the need of specific color measurements. 


\section{ACKN OWLEDGEMENTS}

This research is supported by Grant MAT2009-09795 from the Spanish Ministry of Science and Innovation. A. FernandezOliveras is recipient of a FPU PhD Scholarship from the Spanish Ministry of Science and Innovation. The authors would like to thank Dr. Scott A. Prahl for help in the measurement automation and supervision of the setup used in this work.

\section{References}

[1] J. Qin, and R. Lu, "Measurement of the absorption and scattering properties of turbid liquid foods using hyperspectral imaging," Appl. Spectrosc. 61, 388-396 (2007).

[2] L. L. Randeberg, and L. 0. Svaasand, "Simulated color: a diagnostic tool for skin lesions like port-wine stain," Proc. SPIE 4244, 1-12 (2001).

[3] G .J. Pearson, and K. H., Schuckert, "The role of lasers in dentistry: Present and future," Dent. Update 30, 70-74, 76 (2003).

[4] Y. Shigetani, Y. Tate, A. Okamoto, M. Iwaku, and N. Abu-Bakr, "A study of cavity preparation by Er:YAGlaser. Effects on the marginal leakage of composite resin restoration," Dent. Mater. J. 21, 238249 (2002).

[5] A. H. Jones, A. M. Diaz-Arnold, M. A. Vargas, and D. S. Cobb, "Colorimetric assessment of laser and home bleaching techniques," J. Esthet. Dent. 11, 87-94 (1999).

[6] J. Kato, K. Moriya, J. A. Jayawardena, R. L. Wijeyeweera, and K. Awazu, "Prevention of dental caries in partially erupted permanent teeth with a $\mathrm{CO}_{2}$ laser," J. Clin. Laser Med. Surg 21, 369-374 (2003).

[7] U. Keller, R. Hibst, W. Geurtsen, R. Schilke, D. Heidemann, B. Klaiber, and W. H. Raab, "Erbium:YAG laser application in caries therapy. Evaluation of patient perception and acceptance," J. Dent. 26, 649-656. (1998).

[8] T. M. Ramos, T. M. Ramos-Oliveira, P. M. de Freitas, N. Jr. Azambuja, M. Esteves-Oliveira, N. Gutknecht, and E. C. de Paula, "Effects of Er:YAG and Er,Cr:YSGG laser irradiation on the adhesion to eroded dentin," Lasers Med. Sci. 28, 725-734 (2013).

[9] D.A. Terry, W. Geller, O. Tric, M. J. Anderson, M. Tourville, and A. Kobashigawa, "Anatomical form defines color: function, form and aesthetics," Pract. Proced. Aesthet. Dent. 14, 59-67 (2002).

[10] Y. K. Lee, "Influence of scattering/absorption characteristics on the color of resin composites," Dent. Mater. 23, 124-31 (2007).

[11] Q. Liu, and E. Ruprecht, "Radiative transfer model: matrix operator method," Appl. Opt. 35, 4229-4237, (1996).

[12] S. A. Prahl, "The adding-doubling method" in Optical-thermal response to laser irradiated tissue, A. J. Welch and M. J. C. Ed. van Gemert, 101-129 (New York, 1995).

[13] J. W. Pickering, S. A. Prahl, N. van Wieringen, J. F. Beek, H. J. C. M. Sterenborg, and M. J. C. van Cemert, "Doubleintegrating-sphere system for measuring the optical properties of tissue," Appl. Opt. 32, 399-410 (1993).

[14] S. A. Prahl, M. J. C. van Gemert, and A. J. Welch, "Determining the optical properties of turbid media by using the adding-doubling method," Appl. Opt. 32, 559-568 (1993).

[15] S. A. Prahl iad program (2012): http://omlc.ogi.edu/software/iad/ index.html
[16] L. Wang, S. Sharma, B. Aernouts, H. Ramon, and W. Saeys, "Supercontinuum laser based double-integrating-sphere system for measuring optical properties of highly dense turbid mediain the 1300$2350 \mathrm{~nm}$ region with high sensitivity," Proc. SPIE 8427, 84273B (2012).

[17] D. K. Sardar, B. G. Yust, F. Barrera, L. C. Minum, and A. T. C. Tsin, "Optical absorption and scattering of bovine cornea, lens and retina in the visible region," Lasers Med. Sci. 24, 839-847 (2009).

[18] N. Honda, K. Ishii, A. Kimura, M. Sakai, and K. Awazu, “Determination of optical property changes by laser treatments using inverse adding-doubling method," Proc. SPIE 7175, 71750Q (2009).

[19] K. Ishii, A. Kimura, and K. Awazu, "Optical properties of tissues after laser treatments in the wavelength range of $350-1000 \mathrm{~nm}$," Proc. SPIE 6991, 69912F (2008).

[20] D. K. Sardar, G. Y. Swanland, R. M. Yow, R. J. Thomas and A. T. C. Tsin, "Optical properties of ocular tissues in the near infrared region," Lasers Med. Sci. 22, 46-52 (2007).

[21] Y. C. Chen, J. L. Ferracane, and S. A. Prahl, "A pilot study of a simple photon migration model for predicting depth of cure in dental composite," Dent. Mater. 21, 1075-1086 (2005).

[22] K. Tahir, and C. Dainty, "Experimental measurements of light scattering from samples with specified optical properties," J. Opt. A: Pure Appl. Opt. 7, 207-214 (2005).

[23] S. B. Mitra, D. Wu, and B. N. Holmes, "An application of nanotechnology in advanced dental materials," J. Am. Dent. Assoc. 134, 1382-1390 (2003).

[24] I. Denrya and J. R. Kellyb, "State of the art of zirconia for dental applications," Dent. Mater. 24, 299-307 (2008).

[25] C. Piconi, and G. Maccauro, "Zirconia as a ceramic biomaterial," Biomaterials 20, 1-25 (1999).

[26] J. Chevalier and L. Gremillard, "Ceramics for medical applications: A picture for the next 20 years," J. Eur. Ceram. Soc. 29, 1245-1255 (2009).

[27] A. Fernández-Oliveras, M. Rubiño, and M. M. Pérez, "Scattering anisotropy measurements in dental tissues and biomaterials," J. Europ. Opt. Soc. Rap. Public. 7, 12016 (2012).

[28] A. Fernández-Oliveras, 0. E. Pecho, M. Rubiño, and M. M. Pérez, "Measurements of scattering anisotropy in dental tissue and zirconia ceramic," Proc. SPIE. 8427, 84272C (2012).

[29] A. Fernández-Oliveras, I. M. Carrasco, R. Ghinea, M. Rubiño, and M. M. Pńrez, "Comparison between experimental and computational methods for scattering anisotropy coefficient determination in dental-resin composites," Proc. SPIE. 8427, 84272B (2012).

[30] A. Ishimaru, Wave propagation and scattering in random media (Academic Press, New York, 1978).

[31] S. Chandrasekhar, Radiative Transfer (Dover, New York, 1960).

[32] S. A. Prahl, Light transport in tissue, (PhD thesis, University of Texas, Austin, 1988).

[33] L. C. Andrews, R. L. Philips, Laser beam propagation through random media (SPIE Optical Engineering Press, Bellingham, 2005).

[34] E. Terán, E. R. Méndez, S. Enríquez, and R. Iglesias-Prieto, "Multiple light scattering and absorption in reef-building corals," Appl. Opt. 49, 5032-5042 (2010)

[35] A. Kienle, and M. S. Patterson, "Determination of the optical properties of turbid media from a single Monte Carlo simulation," Phys. Med. Biol. 41, 2221-2227 (1996). 
[36] M. S. Patterson, B. C. Wilson, and D. R. Wyman, “The propagation of optical radiation in tissue $\mathrm{I}$. Models of radiation transport and their application," Lasers Med. Sci. 6, 155-168 (1991).

[37] G. M. Palmer, and N. Ramanujam, "Monte Carlo-based inverse model for calculating tissue optical properties. Part I: Theory and validation on synthetic phantoms," Appl. Opt. 45, 1062-1071 (2006).

[38] G. M. Palmer, C. Zhu, T. M., Breslin, F. Xu, K. W. Gilchrist, and N. Ramanujam, "Monte Carlo-based inverse model for calculating tissue optical properties. Part II: Application to breast cancer diagnosis," Appl. Opt. 45, 1072-1078 (2006).

[39] S. L. Jacques, "Monte Carlo modeling of light transport in tissues" in Optical-thermal response to laser irradiated tissue, A. J. Welch and M. J. C. Ed. van Cemert, (Springer, New York, 1995).

[40] S. A, Prahl, M. Keijzer, S. L. Jacques, and A. J. Welch, "A Monte Carlo model of light propagation in tissue," SPIE Institute Series IS 5, 102-111 (1989).

[41] J. Hourdakis and A. Perris, "A Monte Carlo estimation of tissue optical properties for use in laser dosimetry," Phys. Med. Biol. 40, 351-364 (1995).

[42] J. W. Pickering, C. J. M. Moes, H. J. C. M. Sterenborg, S. A. Prahl, and M. J. C. van Gemert, "Two integrating spheres with an intervening scattering sample," J. Opt. Soc. Am. A 9, 621-631 (1992).

[43] S. A. Prahl, I. A. Vitkin, U. Bruggemann, B. C. Wilson, and R. R. Anderson, "Determination of optical properties of turbid media using pulsed photothermal radiometry," Phys. Med. Biol. 37, 1203-1218 (1991).

[44] International Organization for Standardization, Guide to the Expression of Uncertainty in Measurement., (International Organization for Standardization, Geneva, 1995).

[45] E. Salomatina, B. Jiang, J. Novak, and A. N. Yaroslavsky, "Optical properties of normal and cancerous human skin in the visible and near-infrared spectral range," | Biomed. Opt. 11, 064026-1-9 (2006).
[46] D. K. Sardar, F. S. Salinas, J. J. Perez, and A. T. C. Tsin, "Optical characterization of melanin," J. Biomed. 0pt. 6, 404-411 (2001).

[47] D. K. Sardar, R. M. Yow, A. T. C. Tsin, and R. Sardar, "Optical scattering, absorption and polarization of healthy and neovascularized human retinal tissues," J. Biomed. Opt. 10, 0515011-1-8 (2005).

[48] D. K. Sardar, and L.B. Levy, "Optical Properties of Whole Blood," Lasers Med. Sci. 13, 106-111 (1998).

[49] A. Roggan, H. Albrecht, K. Dörschel, 0. Minet, and G. Müller, "Experimental set-up and Monte-Carlo model for the determination of optical properties in the wavelength range 330-1100 nm," Proc. SPIE 2323, 21-36 (1995).

[50] C. L. Yeh, Y. Miyagawa, and J. M. Powers, "Optical properties of composites of selected shades," J. Dent. Res. 61, 797-801 (1982).

[51] M. Bass, C. DeCusatis, J. Enoch, V. Lakshminarayanan, G. Li, C. MacDonald, V. Mahajan, and E. Van Stryl, Handbook of Optics. Volume IV: Optical Properties of Materials, Nonlinear Optics, Quantum Optics (McGraw Hill Professional, New York, 2009).

[52] E. V. Koblova, A. N. Bashkatov, L. E. Dolotov, Y. P. Sinichkin, T. G. Kamenskikh, E. A. Genina, and V. V. Tuchin, "Monte Carlo modeling of eye iris color," Proc. SPIE 6535, 53521-53521 (2006).

[53] L. L. Randeberg, and L. 0. Svaasand, "Simulated color: a diagnostic tool for skin lesions like port-wine stain," Proc. SPIE 4244, 1-12 (2001).

[54] L. 0. Svaasand, L. T. Norvang, E. J. Fiskerstrand, E. K. S. Stopps, M. W. Berns, and J. S. Nelson, "Tissue parameters determining the visual appearance of normal skin and port wine stains," Lasers Med. Sci. 10, 55-65 (1995). 